DOI: $10.32481 / \mathrm{djph} .2021 .07 .009$

\title{
COVID-19, Preparedness, and Technology:
}

\section{Meeting Access and Functional Needs at Vaccine Points of Dispensing and Beyond}

\author{
Debra Young, MEd, OTR/L, SCEM, ATP, CAPS, FAOTA
}

Disability \& Preparedness Specialist, Association of State and Territorial Health Officials \& Wanderly, Office of Preparedness, Division of Public Health, Delaware Department of Health and Social Services

Technology has become even more important to ensure that the needs of individuals with disabilities and others with access and functional needs (AFN) are met. This is especially apparent in the Delaware Office of Preparedness' planning, mitigation, and response efforts throughout the Coronavirus disease 2019 (COVID-19) pandemic.

The office, based within the Delaware Department of Health and Social Services (DHSS), Division of Public Health (DPH), had already integrated multiple technologies into preparedness planning, mitigation, and recovery efforts prior to the pandemic. It uses the CMIST framework (Communication, Maintaining Health, Independence, Safety/Support Services/SelfDetermination, and Transportation) which encompasses meeting the needs of individuals with disabilities and others with AFN. The framework helps identify individuals' needs before, during, and after an incident. ${ }^{1}$

By using the U.S. Department of Health and Human Services' (HHS) emPOWER data with GIS overlays from the HHS Office of the Assistant Secretary for Preparedness and Response, the Office of Preparedness can locate and support persons who rely on life-maintaining, electricitydependent medical and assistive equipment, such as ventilators, and essential health care services. $^{2}$ There are 7,777 electricity-dependent Medicare beneficiaries in Delaware: 3,617 in New Castle County, 1,601 in Kent County, and 2,559 in Sussex County, according to HHS. Prolonged power outages and other emergencies can be life-threatening for these individuals, who live in their homes. In the event of prolonged outages due to natural disasters, there may be a need for these individuals to be temporarily housed in shelters. These data can assist with possible evacuations and to access alternative power sources.

To better accommodate these Delawareans during such an event, the Office of Preparedness created two kits: an assistive technology (AT) kit and an AFN kit that include purchased equipment. After training DPH's Northern and Southern Health Services personnel on how to use the AT kit, preparedness officials piloted it at multiple small-scale flu vaccine points of dispensing (V-PODs).

With an eye to sustainable program development and an all-hazards approach, the Office of Preparedness envisioned setting up a Functional Assessment Service Team (FAST) to conduct functional assessments and provide support to individuals with disabilities and others with AFN within community shelters and a variety of centers, including heating, cooling, recovery, respite, radiation recovery, community recovery, and/or family assistance centers. As the pandemic unfolded, DPH personnel piloted FAST at COVID-19 mass V-PODs.

Utilizing an equity lens throughout the COVID-19 response was a top priority, and technology ensured equitable vaccine access through the use of low-tech and high-tech accommodations. 
Low-tech accommodations are devices or equipment that do not require much training, may be less expensive, and do not have complex or mechanical features (see figure 1). ${ }^{3}$ High-tech accommodations are described as "the most complex devices or equipment, that have digital or electronic components, and may be computerized." "3 The Office of Preparedness made a variety of low to high-tech accommodations available at the DPH COVID-19 V-PODs for:

- Vision, such as large print materials and magnifiers;

- Hearing, such as portable voice amplifiers, a device to augment hearing aids, a dry erase white board, clear face masks, and Picture Exchange Communication symbols (PECS) and boards;

- Communication, including a Federal Emergency Management Agency website that provides video remote interpretation for American Sign Language (ASL) via the use of a tablet, clear face masks, PECS and boards, and over-the-phone language translation for individuals with limited English proficiency; and

- Mobility, including wheelchairs and walkers.

Figure 1. William Leger, a client of DHSS' Division for the Visually Impaired (DVI), used a signature guide when he came to the Route 9 Library on April 22, 2021 for his second dose of Moderna vaccine. The person with vision loss places their writing instrument in the rectangular cutout area to sign their name in the proper place. Mr. Leger is assisted by Gina Fletcher, a DVI administrative specialist. Source: DVI photo. 


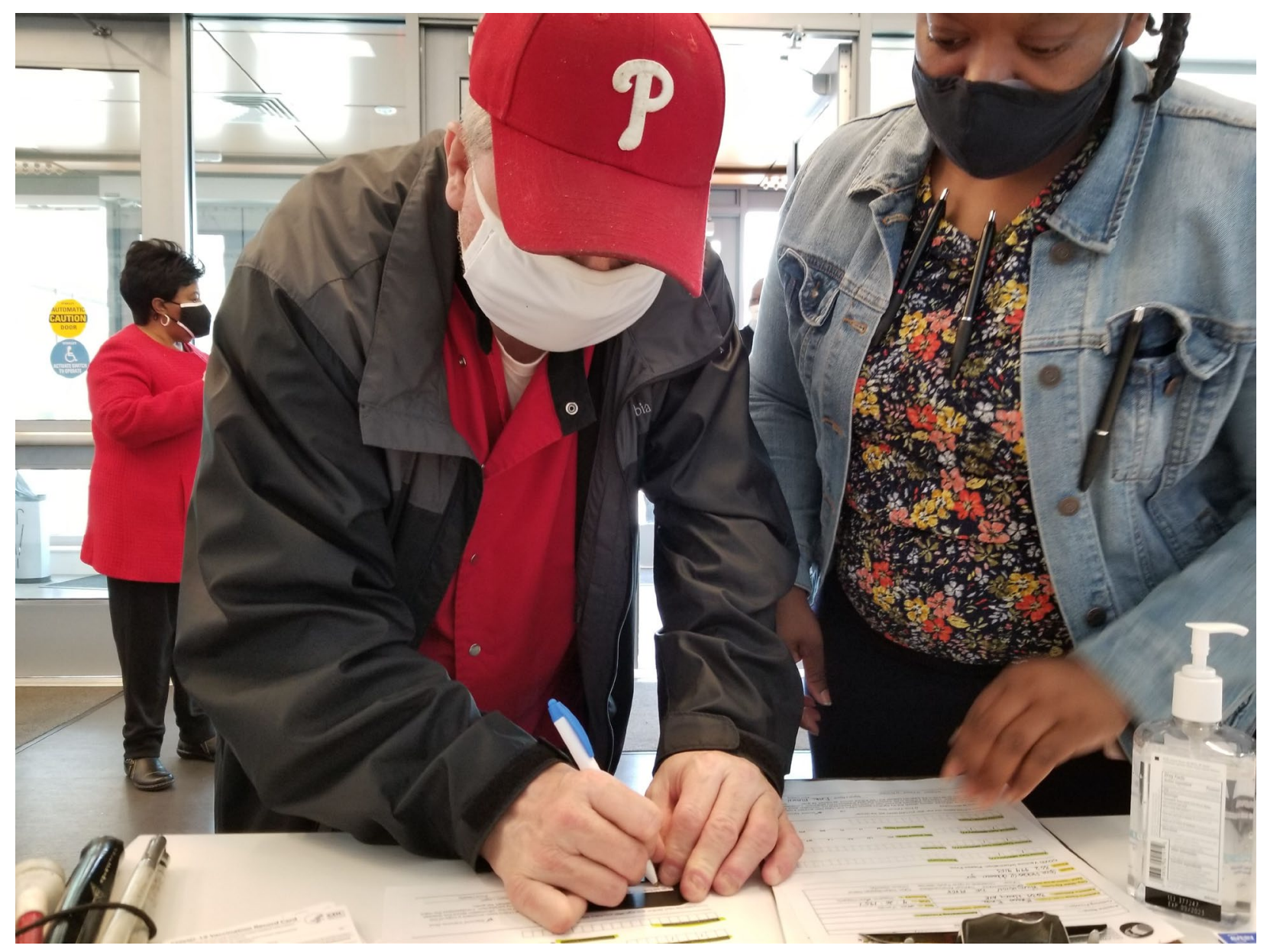

An estimated more than 300 individuals with disabilities and others with AFNs were served at the V-PODs held between January 30, 2021 and June 9, 2021. At one of the mass COVID-19 vaccination events held at the Dover International Speedway, an attendee quickly put his hand up to his ear in an attempt to hear a Delaware National Guard (DNG) member. The DNG member alerted an onsite disability and preparedness specialist from the Association of State and Territorial Health Officials to provide support. Upon assessing the gentleman's needs, the specialist determined that he might benefit from using a voice amplification device. The person requiring the assistive listening support wears headphones, while the other person speaks into the device's microphone and adjusts the volume to meet the individual's needs. Disposable earphone covers are used for hygiene. When the headphones were placed over the gentleman's ears, the specialist asked, "Can you hear me?" The gentleman began to cry joyfully and expressed, "Yes;" he could hear and was grateful that someone cared enough to help him hear better to access the vaccine.

Not all technology needs to be high-tech. There is also a place for low-tech to allow for successful vaccine access. One of the items included in the AT kit is a social story about getting the COVID-19 vaccination. A social story is a social learning tool that supports the safe and meaningful exchange of information between parents, professionals, and people with autism and/or intellectual disabilities of all ages. ${ }^{4}$ The story is illustrated with PECS to present the concepts in clear, simple terms. When a woman and her young adult son arrived at a V-POD, she alerted the DNG that her son had autism. The disability and preparedness specialist provided the 
DOI: $10.32481 /$ djph.2021.07.009

mother with the social story and asked her to read it to her son so he would gain a clear expectation of the vaccination process. He successfully received the vaccine.

To communicate information about the COVID-19 vaccine specifically to meet the needs of individuals with disabilities and others with AFN, the Office of Preparedness has posted much information at https://coronavirus.delaware.gov/vaccine/. The webpage provides communication and clarity surrounding vaccine access, such as V-POD accommodations at vaccination events hosted by the State of Delaware and DPH vendors. Visitors can also find DHSS COVID-19 vaccine initiatives to serve individuals with disabilities and multiple resource links such as a COVID-19 ASL video series and access to Braille and plain language resources. ${ }^{5}$

With its all-hazards approach, the Office of Preparedness continues to utilize technology to support individuals with disabilities and others with AFNs through this crisis and in preparation of future emergencies.

\section{References}

1. U.S. Department of Health \& Human Services Office of the Assistant Secretary for Preparedness and Response (HSS/ASPR). (2020, Aug 27). The CMIST Framework. Public Health Emergency. Retrieved from: https://www.phe.gov/emergency/events/COVID19/atrisk/dischargeplanning/Pages/CMIST-framework.aspx

2. U.S. Department of Health \& Human Services (HHS). (n.d.) HHS emPOWER Program Platform. Retrieved from: https://empowerprogram.hhs.gov

3. Georgia Tech Tools for Life. (n.d.) What is assistive technology? Retrieved from: https://gatfl.gatech.edu/assistive.php

4. Center For Dignity In Healthcare for People With Disabilities. Retrieved from: https://centerfordignity.com/covid-19/

5. Delaware Department of Health and Social Services, Division of Public Health. (n.d.) COVID-19 vaccine information for persons with disabilities or access and functional needs. Retrieved from: https://coronavirus.delaware.gov/vaccine/covid-19-vaccine-informationfor-persons-with-disabilities-or-access-and-functional-needs/

Copyright (c) 2021 Delaware Academy of Medicine / Delaware Public Health Association.

This is an Open Access article distributed under the terms of the Creative Commons Attribution Non-Commercial License (https://creativecommons.org/licenses/by-nc-nd/4.0/) which permits unrestricted non-commercial use, distribution, and reproduction in any medium, provided the original work is properly cited. 